\title{
Configuring Crowdsourcing for Requirements Elicitation
}

\author{
Mahmood Hosseini, Alimohammad Shahri, \\ Keith Phalp, Jacqui Taylor, Raian Ali \\ Bournemouth University, UK \\ \{mhosseini, ashahri, kphalp, jtaylor, rali\}@bournemouth.ac.uk
}

\author{
Fabiano Dalpiaz \\ Utrecht University \\ The Netherlands \\ f.dalpiaz@uu.nl
}

\begin{abstract}
Crowdsourcing is an emerging paradigm which utilises the power of the crowd in contributing information and solving problems. Crowdsourcing can support requirements elicitation, especially for systems used by a wide range of users and working in a dynamic context where requirements evolve regularly. For such systems, traditional elicitation methods are typically costly and limited in catering for the high diversity, scale and volatility of requirements. In this paper, we advocate the use of crowdsourcing for requirements elicitation and investigate ways to configure crowdsourcing to improve the quality of elicited requirements. To confirm and enhance our argument, we follow an empirical approach starting with two focus groups involving 14 participants, users and developers, followed by an online expert survey involving 34 participants from the Requirements Engineering community. We discuss our findings and present a set of challenges of applying crowdsourcing to aid requirements engineering with a focus on the elicitation stage.
\end{abstract}

Keywords-Crowdsourcing, Requirements Elicitation, Crowdbased Elicitation.

\section{INTRODUCTION}

Crowdsourcing is a business model that harnesses the power of a usually large and diverse number of people to contribute knowledge and solve problems [1]. Crowdsourcing is inspired by the need of modern businesses for faster and cheaper solutions. Because of such needs, industrial crowdsourcing platforms have emerged and are being used for real-world problems, e.g., Amazon Mechanical Turk [2] and Threadless [3].

In requirements engineering (RE), it has been shown that crowdsourcing has the potential to aid requirements elicitation [4], [5]. Crowdsourcing facilitates the discovery and involvement of a wider range of stakeholders [6] and users [7]-[10] who can then shape software requirements and the alternative ways for software to fulfil those requirements. Such empowered access increases the volume and enriches the diversity of elicited requirements and, as a result, helps forming a holistic idea of users' and other stakeholders' expectations from a software system. When used iteratively, crowdsourcing refreshes requirements knowledge to reflect how users' requirements evolve and how software should respond [7].

Crowdsourcing systems should be configured to fit tasks that are being solved and also the crowd's and crowdsourcers' characteristics. Crowdsourcing has a variety of definitions, and there has been a need for an agreed and accepted definition for crowdsourcing [11]. However, given the wide variety of views, such a definition is an approximation of putting together the core elements found in the literature. In [12], we observed that the literature still lacks a comprehensive taxonomy of the features that describe crowdsourcing, including the crowd and crowdsourcers. We analysed 113 definitions of crowdsourcing and deduced a taxonomy which represents the space of possible configurations of a crowdsourcing project.

While crowdsourcing has significant potentials for requirements elicitation, we still have little knowledge on how to set up a crowdsourcing project to fit the peculiarities of such a task. Given the large impact of human factors in crowdsourcing, the configuration of crowdsourcing is a difficult task and configuration mistakes could severely affect the quality of elicited requirements. For example, we still have little knowledge on how a large, diverse crowd can assist completeness in requirements and how can such diversity complicate the consensus on the elicited requirements.

The suitability and efficiency of requirements elicitation methods are mainly judged by the quality of requirements that they produce. We may view requirements elicitation as an information system which gathers and organises information and deduces knowledge and design decisions. Requirements quality is therefore a special kind of information quality, and existing frameworks for this purpose, e.g., [13], could be used to measure the quality of requirements. This includes their consistency, completeness, correctness and relevance.

In this paper, we study the configuration of crowdsourcing projects for requirements elicitation. We analyse how the selection of certain crowdsourcing features could affect certain quality dimensions of the elicited requirements. As a method, we analysed the literature first [12] and then followed a mixed methods approach with two phases. In the first, we conducted two focus groups with users and developers. The obtained insights established a baseline for an online expert survey which involved 34 experts requirements engineering (initiated through the REFSQ'14 conference [5]). We discuss our findings and the research challenges related to configuring crowdsourcing for requirements elicitation.

The paper is structured as follows. In Section II. we describe our background work. In Section III] we explain our research methodology. In Section IV], we discuss and reflect on some of the challenges and debates that are introduced as a result of utilising crowdsourcing in requirements elicitation and discuss the threats to the validity of our paper. We conclude the paper in Section $\mathrm{V}$ 


\section{BACKGROUND}

In order to investigate the effects of the utilisation of crowdsourcing features on the process of requirements elicitation, we first separated the human aspects of crowdsourcing and mapped them to those of requirements elicitation. Any crowdsourcing activity consists of four pillars [12], two of which relate to those human aspects. These pillars are the crowd and the crowdsourcer. The crowd in a crowdsourcing activity can be mapped to users and other stakeholders who provide information to requirements engineers, whereas the crowdsourcers in a crowdsourcing activity can be mapped to requirements engineers. This notion has already been confirmed in the empirical track of REFSQ14, where it was first proposed [5]. Table I lists and summarises the extracted features for the crowd and the crowdsourcer from a wide range of crowdsourcing definitions in the existing literature, explained in [12]. The list of features for the crowd and the crowdsourcer was obtained by performing a content analysis on these crowdsourcing definitions. More details on the process of literature study, content analysis and feature extraction are available in our previous study [12].

TABLE I. THE LIST OF CROWD AND CROWDSOURCER FEATURES

\begin{tabular}{lll}
\hline \multicolumn{1}{c}{ The Crowd } & \multicolumn{1}{c}{ The Crowdsourcer } \\
\hline 1. Diversity & 4. Undefined-ness & 1. Incentives Provision \\
1.1. Spatial Diversity & 5. Suitability & 1.1. Financial Incentives \\
1.2. Gender Diversity & 5.1. Competence & 1.2. Social Incentives \\
1.3. Age Diversity & 5.2. Collaboration & 1.3. Entertainment Incentives \\
1.4. Expertise Diversity & 5.3. Volunteering & 2. Open Call \\
2. Unknown-ness & 5.4. Motivation & 3. Ethicality Provision \\
2.1. Not Known to & 5.4.1. Mental & 3.1. Opt-out Provision \\
Crowdsourcer & Satisfaction & \\
2.2. Not Known to & 5.4.2. Self-Esteem & 3.2. Feedback to Crowd \\
Each Other & 5.4.3. Personal & \\
3. Largeness & Skills Development & 3.3. No Harm to Crowd \\
3.1. Number Fulfils & 5.4.4. Knowledge & 4. Privacy Provision \\
the Task & Sharing & \\
3.2. Number Not & 5.4.5. Love of & \\
Abundant & Community & \\
\hline
\end{tabular}

Selection of some of the features of the crowd and the crowdsourcer can potentially influence the quality of the information they provide during requirements elicitation. In order to find the relations between these features and the quality of the information they provide, we obtained insights from the information quality attributes in [13].

\section{Methodology}

To explore the impact of crowdsourcing features on the quality of elicited requirements, we conducted an empirical study following a mixed method sequential exploratory approach [14]. The initial phase was qualitative and consisted of two focus groups with users and developers. The purpose of this phase was to find possible relations between crowdsourcing features in Table $\mathbb{I}$ and quality attributes for elicited requirements. The results were then utilised to form the next quantitative phase of our study, the online expert survey. The purpose of the second phase was to confirm, enhance and explain, via open-ended text, the results we obtained in the first phase.

\section{A. Focus Groups}

The two focus groups were held in two different sessions at Bournemouth University, UK. The same set of questions was used in each session from people with different expertise (Table II). The list of questions we asked in the focus groups from users and developers is available at http://goo.gl/ATs9oq

TABLE II. FOCUS GROUP SESSION SETTINGS

\begin{tabular}{cll}
\hline Session & \multicolumn{1}{c}{ Participants } & \multicolumn{1}{c}{ Expertise } \\
\hline \multirow{2}{*}{1} & Four users and three developers & $\begin{array}{l}\text { All four users are students } \\
\text { Developers are software developers } \\
\text { with three years of experience on average. }\end{array}$ \\
& & $\begin{array}{l}\text { All three users are students } \\
\text { Developers are software developers } \\
\text { with four years of experience on average. }\end{array}$ \\
\hline
\end{tabular}

14 volunteers were invited to participate in the two focus group studies. There were seven participants in each focus group session (Table III). These participants came from ten different countries with mixed backgrounds, different ages and various genders.

Participants in each session were recruited separately through an open call. The same moderator was recruited for both sessions to avoid different attitude towards participants and questions. A questionnaire was also designed and handed over to the participants, which they took time to answer after each interview question and debate. This was mainly done to confirm what the participants expressed during the focus group, and also to allow for the less engaging participants to express their opinions.

The transcribed audio recordings and questionnaire answers from the two focus groups were aggregated and analysed using a content analysis method. The content analysis was performed by two experienced researchers, and if a conflict arose, a third researcher was consulted to investigate and settle the debate. Our focus group study also offered new, interesting discernments on the effects of applying crowdsourcing in a requirements elicitation context.

\section{B. Online Expert Survey}

Based on the results we obtained from the two focus group studies, we identified a set of relations between crowdsourcing features and the quality attributes of elicited requirements. We turned these relations into questions to be given to RE experts to confirm and enhance with further insights. We identified 34 relations in total, classifying them into 10 categories, where each category represented a specific feature of crowdsourcing. These questions were accompanied by nine additional questions which identified the participants by asking questions about their expertise and affiliation in RE, its duration, and their familiarity with crowdsourcing.

The online expert survey was introduced in the opening ceremony of REFSQ'14. The questions in the online expert survey were open-ended, allowing the participants to add their comments and opinions. The complete list of questions we asked from the experts is summarised in Table IIII The participants of the online expert survey were 34 experts from the REFSQ community. The experts' type of expertise in RE, their years of experience in statistics, and their major expertise are summarised in Table IV. 


\begin{tabular}{|c|c|}
\hline Crowdsourcing Feature & $\begin{array}{lc} & \text { List of Items Asked from Experts } \\
\end{array}$ \\
\hline Largeness & $\begin{array}{l}\text { A large crowd supports getting more accurate requirements. } \\
\text { A large crowd supports having objective and non-biased requirements. } \\
\text { A large crowd supports reaching a saturation in the elicited requirements. } \\
\text { A large crowd is difficult to organise and coordinate for eliciting the right requirements. }\end{array}$ \\
\hline Diversity & $\begin{array}{l}\text { Diversity makes it hard to reach a consensus/agreement on requirements. } \\
\text { Diversity increases the relevance and meaningfulness of requirements. } \\
\text { Diversity supports creativity in requirements. } \\
\text { Diversity causes inconsistency problems in elicited requirements. }\end{array}$ \\
\hline Anonymity & $\begin{array}{l}\text { The crowd will give their honest opinion when they are anonymous. } \\
\text { The credibility of the elicited information cannot be guaranteed. }\end{array}$ \\
\hline Competence & $\begin{array}{l}\text { The crowd competence supports getting the right requirements. } \\
\text { The crowd competence supports getting creative requirements. } \\
\text { The crowd competence supports getting more relevant requirements. } \\
\text { A competent crowd is more willing to see positive changes and, hence, willing to provide their requirements. }\end{array}$ \\
\hline Collaboration & $\begin{array}{l}\text { The crowd collaboration means an extra overhead from the management perspective. } \\
\text { The crowd collaboration leads to clusters of users with different and sometimes conflicted views. } \\
\text { The crowd collaboration leads to dominance of certain opinions and missing that of less powerful users. } \\
\text { The crowd collaboration helps requirements engineers to understand the rationale of elicited requirements. }\end{array}$ \\
\hline Intrinsic Motivations & $\begin{array}{l}\text { The crowd motivation supports getting the right requirements. } \\
\text { The crowd motivation supports getting more relevant requirements. } \\
\text { The crowd motivation means that the crowd will give a more complete and detailed answer. }\end{array}$ \\
\hline Volunteering & $\begin{array}{l}\text { A volunteering crowd is more likely to state their true and genuine requirements. } \\
\text { Open calls provide a chance for malicious users to enter the elicitation process and affect the overall quality of elicited requirements. }\end{array}$ \\
\hline Extrinsic Incentives & $\begin{array}{l}\text { Incentives motivate the crowd to be more active during requirements elicitation. } \\
\text { Incentives increase the number of participants. } \\
\text { Incentives mislead the crowd from acting truly on requirements elicitation. }\end{array}$ \\
\hline Opt-out Opportunity & $\begin{array}{l}\text { Providing an opt-out opportunity motivates the participants for active involvement. } \\
\text { Providing an opt-out opportunity attracts more participants. } \\
\text { Providing an opt-out opportunity allows only motivated participants to carry on to the end, which means an improved quality of the elicited requirements. }\end{array}$ \\
\hline Feedback & $\begin{array}{l}\text { Feedback motivates the participants to engage. } \\
\text { Feedback disturbs participants' comfort. } \\
\text { Feedback could influence their opinion for the next stages. } \\
\text { Feedback gives participants the feeling that their ideas are important. } \\
\text { Feedback increases the willingness of participants to participate in future studies. }\end{array}$ \\
\hline
\end{tabular}

TABLE IV. SUMMARY OF EXPERTS' INFORMATION

\begin{tabular}{lclcl}
\hline \multicolumn{2}{c}{$\begin{array}{c}\text { Types of } \\
\text { Expertise in RE }\end{array}$} & \multicolumn{2}{c}{$\begin{array}{c}\text { Years of } \\
\text { Expertise in RE }\end{array}$} & \multicolumn{1}{c}{ Major Expertise in RE } \\
\hline Academic & 18 & Min & 2 & User-Centred RE, Collaborative RE, \\
& & Max & 33 & Modelling, Management, Specification, \\
Industrial & 7 & Mean & 9.44 & Traceability, Goal Oriented RE, \\
& & Median & 8 & Privacy and Security Requirements, \\
Both & 9 & Mode & 10 & Social RE, Automation for RE \\
\hline
\end{tabular}

After closing the online expert survey, all the provided data, including the comments that the experts had provided, was aggregated and analysed using both quantitative and qualitative methods. Based on this analysis, we identified a list of challenges in utilising crowdsourcing in the process of requirements elicitation. These challenges are presented in the next section.

\section{Challenges}

In this section, we discuss challenges and issues that our study illustrates to be important for further investigation in order to successfully develop and apply crowdsourcing for requirements elicitation. These challenges are informed by our review of the literature, e.g., in [12] and [15] and the results of the studies conducted in this paper, including the points which were debatable amongst the experts and the users. Other challenges are based on our arguments which is also supported by the literature in other domains like psychology and sociology. We further elaborate on these challenges as follows.

\section{A. List of Challenges}

1) Challenges Related to Largeness: On one hand, the large crowd is acknowledged to maximise accuracy, relevance and saturation in elicited requirements. Furthermore, while it has been shown that there are no simple analytical procedures to decide whether end-users have stated all their requirements to software developers [16], involving a large group of users can be seen as a way to minimise this problem. On the other hand, however, it also has its pitfalls related to management and coordination. As requirements engineering is generally viewed as a collaborative activity [17], coordination becomes an issue when requirements engineers have to deal with a large group of users during the RE process. Developing softwarebased mechanisms to coordinate the crowd with minimum intervention from developers, and to stay cost-efficient, is a challenge. At the same time, the strictness of such platforms should not violate the principles of crowdsourcing which basically advocate the voluntary nature of the participation.

2) Challenges Related to Diversity: Experts highly agreed on the benefits of diversity in leading to more relevant and creative requirements. However, it is not cost-free. Diversity might introduce problems in reaching an agreement, especially when the software should serve diverse sets of users and roles. Geographical diversity, in particular, has been shown to cause several problems in the field of RE, namely inadequate communication, difficulties in knowledge management, challenges in cultural diversity and issues related to time differences [18]. Aggregation of knowledge from a wide set of users and identifying potential decisions is a challenge too. Current widely accepted visions, like Wisdom of Crowds [19] and Swarm Intelligence [20], still do not offer systematic ways to such a human-driven aggregation and coordination. The 
literature in communities like AI and agent-based computing provides deals mainly with artificial computation units (agents) while human-factors are not a main focus.

3) Challenges Related to Anonymity: Anonymity typically makes users more honest in discussing their opinions. For example, it is argued that in the 360-degree literature, anonymity enhances honesty in ratings [21]. In another study, anonymity was related to improved quality and quantity of comments in an online learning community [22]. Furthermore, anonymity is one way of assuring users' privacy and security, as is discussed in [23]. However, it can also be risky as it would allow malicious users to join in. Consequently, anonymity is not allowed in some businesses being crowdsourced and also not allowed due to data protection rules and intellectual properties in certain environments. Furthermore, anonymity might discourage users who care more about social recognition and would like to see their voices heard and get recognition for that. For example, [22] found that online identity and reputation were more important in non-anonymous groups, compared to anonymous groups. Finally, even though anonymity is not associated with antisocial behaviour per se, but it has been shown to contribute to deindividualisation, which is a state of loss of self-awareness, decreased social disinhibitions, and increased impulsivity [24]. Such an adverse effect of anonymity may also affect users' social stance and lead to aggressive behaviour and dishonest conduct [24]. Managing anonymity in a way which considers these points is a challenge. One way that is being used in research investigating the trustworthiness of online reviews is the use of validated sources.

4) Challenges Related to Competence: It appears that high competence in the crowd is always positive with no negative consequences. As a result, finding the right crowd with the required competencies has been the subject of many crowdsourcing studies [25], [26]. However, the reality could sometimes be different. Crowd work has shown the potential to replace some forms of skilled labour with unskilled labour, since crowdsourced tasks are usually divided into smaller tasks, called micro tasks [27]. Literature also shows that some complex tasks and tasks that require expertise, such as product design and translation can also be amenable to novice crowd workers with appropriate process design and technological support [28], [29]. Furthermore, for creative requirements, differences in the competence level could be desirable to stimulate new ideas [30], [31] and also because the final system typically serves both competent and less competent users. This links with our discussion of diversity earlier. Furthermore, recruiting the competent crowd might mean additional financial costs, which may lead to restrictions in the number of participants, i.e., crowd largeness. The competent crowd might also include participants' inflated egos which would then reduce the level of collaboration and lead to conflicts and inconsistency. Consequently, choosing the degree and variety of competence level and managing those trade-offs remain among the main challenges.

5) Challenges Related to Collaboration: Software engineering projects are inherently collaborative, since there are many stakeholders involved [32]. Collaboration benefits are many, such as the ability to realise the rationale for requirements and having holistic solutions. The challenges are outnumbered by the benefits and mainly relate to the organi- sation and ensuring that clustering and dominance of certain opinions, trends, and groups will not emerge. To address these challenges, some studies have been done independently in order to find engineering solutions for collaboration [33]. For example, it has been illustrated in [34] that independence, individuality, capability, sustainability and leanness are properties of successful collaborative environments. Other cross-cutting challenges include how collaboration will be implemented with anonymity and how incentives will be offered when the work is done collaboratively. When decisions are being made as to whether to participate in a request for feedback, this frequently links to personality, e.g., whether they are more collaborative or competitive, and this in turn can be related to intrinsic and extrinsic motivation.

6) Challenges Related to Intrinsic Motivations: Intrinsic motivation in human beings is a pervasive and important form of motivation which is considered to be a critical element in physical, social, and cognitive development of humans [35]. Intrinsically motivated participants are genuinely interested in the software for which requirements are crowdsourced and thus give better quality information. Although the effects of intrinsic motivations of the participation of the crowd has been extensively studied in the domain of crowdsourcing [36], [37], it is still difficult to identify metrics and tests for such an attribute in participants. Also, motivation may lead to bias and strong views on what requirements the system should fulfil and could, thus, affect collaboration and reaching agreement.

7) Challenges Related to Volunteering: Volunteering is sometimes regarded as a core element of crowdsourcing, and other times it is regarded as the opposite of work for pay [38]. Either way, challenges in other dimensions, such as anonymity, incentives and competence, sometimes overlap with volunteering challenges. For example, recruiting volunteers and offering incentives must be done cautiously to prevent volunteers from getting demotivated over time. Furthermore, competence of volunteers should be taken into consideration during recruitment to avoid reduced quality.

8) Challenges Related to Extrinsic Incentives: Extrinsic incentives are generally more prevalent in humans' lives as they are associated with social demands and roles that require individuals to assume responsibility for non-intrinsically interesting tasks [35]. Providing such incentives can mean higher costs or more efforts on the crowdsourcer's part and does not necessarily mean more reliable requirements [39]. Furthermore, extrinsic incentives can harm intrinsic motivations in users and thus affect the quality of the elicited requirements as well. This is one of the reasons for many studies on the effects of extrinsic incentives on intrinsic motivations [40], [41]. Finding the right incentives and how it is linked to competence, intrinsic motivation and anonymity are other research challenges to investigate.

9) Challenges Related to Opt-out Opportunity: Providing the opportunity for participants to opt out at any time is considered to be in the core of crowdsourcing, which is typically seen as a loose contractual model based on voluntary participation. As it was the case with volunteering, challenges in other dimensions sometimes overlap with opt-out challenges. For example, it is shown in [42] that inadequate incentives or higher complexity of tasks may lead participants to opt out. This suggests that there are causal relationships to explore 
among these factors and the possibility and desire of the crowd to opt-out.

10) Challenges Related to Feedback: Providing feedback to the crowd is often seen in a positive way. This is especially the case when the feedback is provided in a timely manner and is task-specific [43], which can improve the performance of participants and also motivate them to persevere and accept more tasks. However, we still need to investigate how to decide what feedback to give and when to do that in a way that it does not affect participants' opinion for the next steps and, also, does not overload them with unnecessary information. Since feedback has a social influence on the participant, it can lead to convergence of opinions [44] and elimination of diversity of opinions, which produces adverse effects on the elicited requirements.

\section{B. Threats to Validity}

Our empirical study involved 34 experts in order to confirm the first phase observations and to see what debates could arise. There was also a balance in terms of academic and industrial expertise. The questions were appended by text boxes to add further insights, which explained their choices in most cases. The questions were developed based on two focus groups including both users and developers so that we ensured they are relevant. In spite of that, our study still has few threats to validity:

- The online expert survey consisted of an online questionnaire with text boxes for further comments, and was introduced to REFSQ14 community, which is principally a requirements engineering community. Crowdsourcing, however, is a multi-disciplinary topic and our obtained results may reflect only the opinions of requirements engineers with technical expertise in classical areas in the domain such as elicitation, traceability and specification. However, this threat is partly alleviated as our main focus of the study was also on the application of crowdsourcing for requirements elicitation.

- Crowdsourcing for RE is a relatively new area. In our study, some experts stated they could not find research and tools for it. For these experts, this could mean that their answers on how crowdsourcing would aid elicitation were speculative. However, we consider that their answers are still valid especially because the elements of crowdsourcing that they were asked about in our study could also be found in traditional elicitation, e.g., the diversity of users to elicit requirements from and their competence.

- $\quad$ Our work studies the effects of crowdsourcing features individually on the quality of requirements. This limited the accuracy of the results as feature effects could sometimes differ in the existence of other features, e.g., the effect of largeness on correctness might be affected whether the crowd is competent or not.

- $\quad$ There could be other relations between crowdsourcing features and requirements quality. The ones we studied in this paper are those which emerged from our two focus groups.

\section{CONCLUSIONS}

Considering the lack of knowledge on how crowdsourcing should be configured to maximise the quality of elicited requirements, we conducted this study which consisted of two focus groups and an online expert survey. The study allowed us to deduce and confirm a set of relations among crowdsourcing features and requirements quality. Based on experts' opinions, we advocated that there is a huge potential of crowdsourcing for requirements elicitation and observed that there is not a significant amount of literature investigating it. We also observed that such use of crowdsourcing introduces new research problems and a wide range of trade-offs, which makes the decision with adopting it and configuring it in the correct way challenging. Our paper is meant to provide insights for researchers and practitioners in the field towards a crowdcentred requirements and systems engineering where the crowd is given a voice in the engineering process.

Our future work includes a detailed analysis of the data we have gathered from the focus groups and the online expert survey. The analysis of this data will shed more light on the benefits and challenges of the utilisation of crowdsourcing in the process of requirements elicitation. It will also help requirements engineers in finding the right configuration of crowdsourcing features for the ultimate purpose of system design and evolution. We also aim to take the study further and find the inter-relations between crowdsourcing features and how these inter-relations may affect the quality of requirements.

\section{ACKNOWLEDGMENT}

The research was supported by a European FP7 Marie Curie CIG grant (the SOCIAD Project) and Bournemouth University through the Graduate School Santander Grant for PGR Development.

\section{REFERENCES}

[1] J. Howe. (2006, June) Crowdsourcing: A definition. [Online]. Available: http://www.crowdsourcing.com/cs/2006/06/crowdsourcing_a.html

[2] P. G. Ipeirotis, "Analyzing the amazon mechanical turk marketplace," XRDS: Crossroads, The ACM Magazine for Students, vol. 17, no. 2, pp. 16-21, 2010.

[3] J. Wu, C. Damminga, K. K. P. Johnson, and H.-Y. Kim, "Content analysis of online co-design community interactions: A case study of crowd-sourced threadless," Journal of Global Academy of Marketing Science, vol. 20, no. 4, pp. 334-342, 2010. [Online]. Available: http://dx.doi.org/10.1080/12297119.2010.9707437

[4] A. Adepetu, A. Khaja, Y. A. Abd, A. A. Zaabi, and D. Svetinovic, "Crowdrequire: A requirements engineering crowdsourcing platform," in Proceedings of the 2012 AAAI Spring Symposium: Wisdom of the Crowd, 2012.

[5] M. Hosseini, K. Phalp, J. Taylor, and R. Ali, "Towards crowdsourcing for requirements engineering," in Proceedings of the 20th International working conference on Requirements engineering: foundation for software quality(REFSQ)-Empirical Track, 2014.

[6] S. L. Lim, D. Quercia, and A. Finkelstein, "Stakenet: Using social networks to analyse the stakeholders of large-scale software projects," in Proceedings of the 32nd ACM/IEEE International Conference on Software Engineering (ICSE)-Volume 1. ACM, 2010, pp. 295-304.

[7] R. Ali, C. Solis, I. Omoronyia, M. Salehie, and B. Nuseibeh, "Social adaptation: when software gives users a voice," in Proceedings of the 7th International Conference on Evaluation of Novel Approaches to Software Engineering (ENASE), June 2012. 
[8] R. Ali, C. Solis, M. Salehie, I. Omoronyia, B. Nuseibeh, and W. Maalej, "Social sensing: When users become monitors," in Proceedings of the 19th ACM SIGSOFT symposium and the 13th European conference on Foundations of software engineering (ESEC/FSE), 2011, pp. 476-479.

[9] D. Pagano and B. Brügge, "User involvement in software evolution practice: A case study," in Proceedings of the 2013 international conference on Software engineering (ICSE). Piscataway, NJ, USA: IEEE Press, 2013, pp. 953-962.

[10] D. Pagano and W. Maalej, "User feedback in the appstore: An empirical study," in Proceedings of the 2013 IEEE Requirements Engineering Conference, 2013, pp. 125-134.

[11] E. Estellés-Arolas and F. González-Ladrón-de Guevara, "Towards an integrated crowdsourcing definition," Journal of Information science, vol. 38 , no. 2, pp. 189-200, 2012.

[12] M. Hosseini, K. Phalp, J. Taylor, and R. Ali, "The four pillars of crowdsourcing: A reference model," in Proceedings of the 2014 IEEE Eighth International Conference on Research Challenges in Information Science (RCIS), 2014, pp. 1-12.

[13] B. K. Kahn, D. M. Strong, and R. Y. Wang, "Information quality benchmarks: Product and service performance," Communications of the $A C M$, vol. 45, no. 4, pp. 184-192, 2002.

[14] J. W. Creswell and V. L. P. Clark, Designing and conducting mixed methods research. SAGE Publications, Inc, 2011.

[15] M. Hosseini, K. Phalp, J. Taylor, , and R. Ali, "On the configuration of crowdsourcing projects," International Journal of Information System Modeling and Design (IJISMD), 2015.

[16] G. Kotonya and I. Sommerville, "Requirements engineering with viewpoints," Software Engineering Journal, vol. 11, no. 1, pp. 5-18, 1996.

[17] L. Macaulay, "Requirements capture as a cooperative activity," in Proceedings of 1993 IEEE International Symposium on Requirements Engineering. IEEE, 1993, pp. 174-181.

[18] D. E. Damian and D. Zowghi, "The impact of stakeholders' geographical distribution on managing requirements in a multi-site organization,' in Proceedings of 2002 IEEE Joint International Conference on Requirements Engineering. IEEE, 2002, pp. 319-328.

[19] J. Surowiecki, The wisdom of crowds. Random House LLC, 2005.

[20] E. Bonabeau, M. Dorigo, and G. Theraulaz, Swarm intelligence. Oxford, 1999.

[21] J. Ghorpade, "Managing five paradoxes of 360-degree feedback," The Academy of Management Executive, vol. 14, no. 1, pp. 140-150, 2000.

[22] P. G. Kilner and C. M. Hoadley, "Anonymity options and professional participation in an online community of practice," in Proceedings of th 2005 conference on Computer support for collaborative learning: learning 2005: the next 10 years! International Society of the Learning Sciences, 2005, pp. 272-280.

[23] A. Beach, M. Gartrell, and R. Han, "Solutions to security and privacy issues in mobile social networking," in Proceedings of the 2009 International Conference on Computational Science and Engineering (CSE), vol. 4. IEEE, 2009, pp. 1036-1042.

[24] M. Kabay, "Anonymity and pseudonymity in cyberspace: deindividuation, incivility and lawlessness versus freedom and privacy," in Proceedings of the 1998 Annual Conference of the European Institute for Computer Anti-virus Research (EICAR), vol. 16, 1998, p. 8.

[25] L. Erickson, I. Petrick, and E. Trauth, "Hanging with the right crowd: Matching crowdsourcing need to crowd characteristics." AIS Electronic Library (AISeL), 2012.

[26] C. Wiener, I. N. Acquah, M. Heiss, T. Mayerdorfer, M. Langen, and W. C. Kammergruber, "Targeting the right crowd for corporate problem solving-a siemens case study with technoweb 2.0," in Proceedings of the 2012 IEEE International Technology Management Conference (ITMC). IEEE, 2012, pp. 239-247.
[27] A. Kittur, J. V. Nickerson, M. Bernstein, E. Gerber, A. Shaw, J. Zimmerman, M. Lease, and J. Horton, "The future of crowd work," in Proceedings of the 2013 Conference on Computer Supported Cooperative Work (CSCW). New York, NY, USA: ACM, 2013, pp. 1301-1318.

[28] A. Kittur, B. Smus, S. Khamkar, and R. E. Kraut, "Crowdforge: Crowdsourcing complex work," in Proceedings of the 24th annual ACM symposium on User interface software and technology. ACM, 2011, pp. 43-52.

[29] A. Kittur, "Crowdsourcing, collaboration and creativity." ACM Crossroads, vol. 17, no. 2, pp. 22-26, 2010.

[30] L. Yu and J. V. Nickerson, "Cooks or cobblers?: crowd creativity through combination," in Proceedings of the 2011 SIGCHI conference on human factors in computing systems. ACM, 2011, pp. 1393-1402.

[31] L. yu and J. V. Nickerson, "An internet-scale idea generation system," ACM Transactions on Interactive Intelligent Systems (TiiS), vol. 3, no. 1, pp. 2:1-2:24, April 2013.

[32] J. Whitehead, "Collaboration in software engineering: A roadmap," in 2007 Future of Software Engineering (FOSE), ser. FOSE '07. Washington, DC, USA: IEEE Computer Society, 2007, pp. 214-225.

[33] R. Klinc, Z. Turk, and M. Dolenc, "Engineering collaboration 2.0: requirements and expectations," 2009.

[34] Z. Turk, T. Cerovsek, and R. J. Scherer, "Issues in integration of civil engineering services and tools." in Proceedings of the 2003 International Conference on Internet Computing, 2003, pp. 219-224.

[35] R. M. Ryan and E. L. Deci, "Intrinsic and extrinsic motivations: Classic definitions and new directions," Contemporary Educational Psychology, vol. 25 , no. 1 , pp. $54-67,2000$.

[36] M. Hossain, "Users' motivation to participate in online crowdsourcing platforms," in Proceedings of the 2012 International Conference on Innovation Management and Technology Research (ICIMTR). IEEE, 2012, pp. 310-315.

[37] J. Rogstadius, V. Kostakos, A. Kittur, B. Smus, J. Laredo, and M. Vukovic, "An assessment of intrinsic and extrinsic motivation on task performance in crowdsourcing markets." in Proceedings of the 2011 International AAAI Conference on Web and Social Media (ICWSM), 2011.

[38] A. Mao, E. Kamar, Y. Chen, E. Horvitz, M. E. Schwamb, C. J. Lintott, and A. M. Smith, "Volunteering versus work for pay: Incentives and tradeoffs in crowdsourcing," in Proceedings of the First AAAI Conference on Human Computation and Crowdsourcing, 2013.

[39] M. Varela, T. Mäki, L. Skorin-Kapov, and T. Hoßfeld, "Increasing payments in crowdsourcing: dont look a gift horse in the mouth," in Proceedings of the 4th international workshop on perceptual quality of systems (PQS 2013), Vienna, Austria, 2013.

[40] E. L. Deci, R. Koestner, and R. M. Ryan, "A meta-analytic review of experiments examining the effects of extrinsic rewards on intrinsic motivation." Psychological bulletin, vol. 125, no. 6, p. 627, 1999.

[41] C. D. Fisher, "The effects of personal control, competence, and extrinsic reward systems on intrinsic motivation," Organizational Behavior and Human Performance, vol. 21, no. 3, pp. 273-288, 1978.

[42] B. Shao, L. Shi, B. Xu, and L. Liu, "Factors affecting participation of solvers in crowdsourcing: an empirical study from china," Electronic Markets, vol. 22, no. 2, pp. 73-82, 2012.

[43] S. Dow, A. Kulkarni, B. Bunge, T. Nguyen, S. Klemmer, and B. Hartmann, "Shepherding the crowd: managing and providing feedback to crowd workers," in Proccedings of the CHI'11 Extended Abstracts on Human Factors in Computing Systems. ACM, 2011, pp. 1669-1674.

[44] J. Lorenz, H. Rauhut, F. Schweitzer, and D. Helbing, "How social influence can undermine the wisdom of crowd effect," Proceedings of 2011. 